\title{
Validity and Reliability of Posture Screen Mobile Application in Adolescent Idiopathic Scoliosis
}

\author{
NASR A.A. OTHMAN, Ph.D.**; AHMED S.A. ASKER, M.Sc.*; SALWA F. ABD ELMAGEED, Ph.D.** and \\ NAGY A. SABET, M.D.*** \\ The Department of Physical Therapy for Musculoskeletal Disorders and its Surgeries, Faculty of Physical Therapy, \\ Badr* \& Cairo** Universities and Department of Orthopedic Surgery, Faculty of Medicine, \\ Misr University for Science \& Technology, Giza***, Egypt
}

\begin{abstract}
Background: Idiopathic scoliosis is a three-dimensional deformity of the growing spine, affecting $2 \%-3 \%$ of adolescents. Curve detection before skeletal maturation gives a good opportunity for early treatment or prevention of curve progression.

Aim of Study: To investigate validity and reliability (intrarater) of posture screen mobile (PSM) application in Adolescent idiopathic scoliosis (AIS).

Material and Methods: Forty-one subjects (28 females \& 13 males) with idiopathic scoliosis ( 30 thoracolumbar \& 11 lumbar) participated in this study. Their mean \pm SD for age, weight, height, and BMI were $17( \pm 1.2)$ years, 65.97 $( \pm 9.16) \mathrm{kg}, 168.6( \pm 6.4) \mathrm{cm}$ and $23.1( \pm 2.04) \mathrm{kg} / \mathrm{m}^{2}$ respectively. Cobb's angles for each patient were predetermined by the radiologist before participating in this study. Each patient was clinically scanned by using two cameras (mobile phone $\&$ IPad) from four views (anterior, posterior, right lateral, left lateral). Following digitization, the application calculates the translations and angulations of different spinal segments.
\end{abstract}

Results: Moderate positive significant correlation for T1$\mathrm{T} 4$ ( $r=0.4, p=0.009)$, and strong positive significant correlation for T4-T8, T8-T12, T12-L3 and L3-PSIS ( $r=0.82$ to 0.89 ), $p=0.0001$ ) were found between Cobb's angle and spinal segment angulation measured by posture screen mobile. On the other hand moderate positive significant correlation for T1-T4 ( $r=0.6, p=0.009$ ), and strong positive significant correlation for T4-T8, T8-T12, T12-L3 and L3-PSIS ( $r=0.8-0.9$ ), $p=0.0001$ ) were found between Cobb's angle and segment translation that measured by posture screen mobile.Intra-rater reliability of the Posture screen mobile suggested excellent agreement for spinal segment angulation (ICC: 0.99) and spinal segments translation (ICC: 0.92-0.97).

Conclusion: PSM application is valid and reliable in screening posture deviations for patients with idiopathic scoliosis. It is an easy, quick, safe, affordable, user-friendly, low-cost screening method, and noninvasive for clients and patients.

Key Words: Scoliosis - Cobb's angle-Posture screen mobile.

Correspondence to: Dr. Nasr A.A. Othman
E-Mail: dr.nasrawad@cu.edu.eg
dr.nasrawad@gmail.com

Correspondence to: Dr. Nasr A.A. Othman dr.nasrawad@gmail.com

\section{Introduction}

SCOLIOSIS is defined as a lateral curvature of the spine greater than 10 degrees using Cobb's angle on posteroanterior (PA) radiographs [1] Adolescent idiopathic scoliosis (AIS) is a common disease that occurs at the age of 11-18 years with an overall prevalence of $0.47-5.2 \%$. Approximately $90 \%$ of idiopathic scoliosis develops during the childhood period [2]. The accurate assessment of scoliosis requires an erect anteroposterior radiograph of the full spine. From the AP image, the internal bony configuration and the extent of lateral deviation of the spine can be determined. Various measurements such as those of pedicle rotation may be taken from the radiograph. However, the technique most advocated by the Scoliosis Research Society has been Cobb's method. When using Cobb's method, an angle that quantifies the extent of lateral curvature within the affected region of the spine is calculated. Measurements obtained with Cobb's method do not fully describe the threedimensional geometry of the spine and associated deviations, such as axial rotation [3].

Currently, the standard care for AIS includes observation for the patients with small curves or skeletal maturity, brace treatment for those with moderate curves and skeletal immaturity, and operation for those with severe curves [4]. The effectiveness of treatment in persons with idiopathic scoliosis has been criticized and this may be due to the lack of adequate clinical measurement tools to monitor objective change in body posture. Radiographs are invasive methods when used for repeated measures to follow-up scoliosis. Several 3D posture analysis systems (such as Vicon, Opti Track, Motion analysis, and Surface topography) are used to assess posture. However, these systems 
are not easily accessible for most clinicians as they are very expensive and require specialized trained persons to deal with their complex software. Digital imaging has many advantages in terms of convenience, portability, and ability to adjust contrast, brightness, and magnification, leading to increased accuracy of measurements compared to manual methods [5].

PostureScreen Mobile (PSM) is a relatively new device that can effectively assess posture in a variety of settings. It is designed for many professionals to screen subjects for postural deviations. It is a quick, affordable, noninvasive, and easyuse tool. The PSM application can be installed on portable devices, such as an iPad. Using front, back and side-view photographs of the subject, the PSM directs the user in the identification of anatomical landmarks for a rapid assessment of posture [6]. Thus, this study aimed to provide scientific evidence on the validity and reliability of posture screen mobile application in measuring posture deviation in patients with AIS.

\section{Patients and Methods}

This cross-sectional study was conducted at the outpatient clinic, Faculty of Physical Therapy, Cairo University from July 2018 to March 2019. To avoid type II error, sample size calculation was performed prior to the study using G*POWER statistical software (version 3.1.9.2; Franz Faul, Universitat Kiel, Germany) [Exact tests- correlational study, $\alpha=0.05, \beta=0.2$, and large effect size $=0.4 \mathrm{I}$ and revealed that the appropriate sample size for this study was $\mathrm{N}=41$.

\section{Patients:}

A total of 50 patients were assessed for eligibility criteria as shown in Fig. (1). Nine patients were excluded. Two because they did not meet the inclusion criteria of this study and seven refused to participate in the study. A total of 41 patients (28 females and 13 males) with idiopathic scoliosis participated in this study. Their mean \pm SD age, weight, height, and BMI were $17( \pm 1.2)$ years, $65.97( \pm 9.16) \mathrm{kg}, 168.6( \pm 6.4) \mathrm{cm}$, and $23.1( \pm 2.04)$ $\mathrm{kg} / \mathrm{m}^{2}$ respectively.

Patients who had (1) idiopathic scoliosis with mild to moderate degree $\left(10^{\circ}-45^{\circ}\right),(2)$ age range from 11 to 18 years, and (3) $\mathrm{BMI}=20-25 \mathrm{~kg} / \mathrm{m}^{2}$ were included in the study. Patients were excluded if they had severe idiopathic scoliosis (greater than $45^{\circ}$ ), congenital deformities (leg length discrepancy), musculoskeletal spinal disorders other than scoliosis (hyper kyphosis \& hyper lordosis), structural defects of the spinal vertebra (hemivertebra), BMI $>25 \mathrm{~kg} / \mathrm{m}^{2}$, previous spinal surgery, and associated pathologies that may interfere with maintaining an erect standing posture such as cerebellar or inner ear disorders.

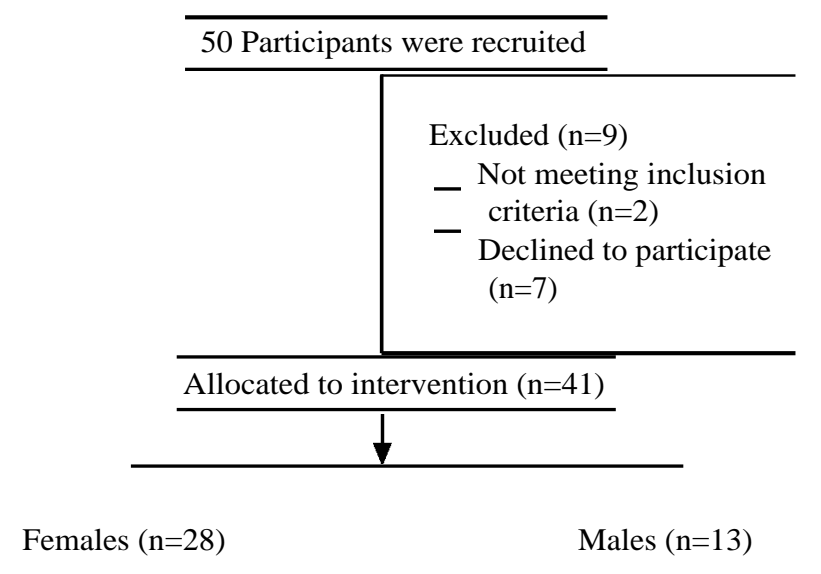

Fig. (1): Participant's flowchart.

\section{Measurement procedures:}

All subjects were familiarized with the procedure by explanation and demonstration. Testing procedures were completed in an isolated room. Written consent for acceptance to share in this study was signed by everyone. Patient name, date of birth, height, and weight was entered into PSM as a basic requirement. Patients were asked to wear minimal clothing (shorts for men, and shorts \& bra or tight clothes for women) also with their shoes removed. All patients were assessed by the same examiner.

Full spine PA X-ray for all patients were done and Cobb's angle was measured by radiologist digitally and double-check was done manually by another two examiners to confirm the obtained Cobb's angle degree. For each patient, reflecting dots (markers) according to the system software were placed on a specific spinous process by an experienced orthopedic physical therapist. Eleven landmarks were placed on the posterior view image at the following locations: Right acromion, left acromion, spinous process of $\mathrm{T} 1$, spinous process of $\mathrm{T} 4$, spinous process of $\mathrm{T} 8$, spinous process of $\mathrm{T} 12$, spinous process of L3, right posterior superior iliac spine (PSIS), left PSIS, left rib-T8 level, right rib-T8 level (Fig. 2A). Five landmarks were placed on the lateral view image at the following locations: tragus, the acromioclavicular joint, greater trochanter, lateral femoral condyle, and lateral malleolus of the ankle joint (Fig. 2C). Nine landmarks were placed on the anterior view image at the following 
locations: top of the right acromioclavicular (AC) joint, episternal notch, top of the left AC joint, right lateral rib-T8 level, left lateral rib-T8 level, right anterior superior iliac spine (ASIS), left ASIS, the center of the right talus, center of the left talus (Fig. 2B). Each patient was clinically scanned with mobile phone (iPad with IOS operating system and a 12-megapixel camera), which fixed to a tripod that was placed on a stand exactly 10 feet away from the subject markers at a height of 4.5 feet to standardize the image angle.

The following instructions were given to all patients: "take a deep breath, place your hands at your side, relax and stand normally". The patients were also instructed to keep their weight evenly distributed over both feet. Four digital photographs were taken for the posterior, lateral (right $\&$ left), anterior views. When taking the picture, the PSM application shows a target-like display that turns green when the tablet is level, this ensures that each image is taken at a level and consistent angle. Once each picture is captured, the PSM application takes the user through the process of digitizing specified anatomical landmarks in order to produce anterior, posterior, lateral translations, and angular

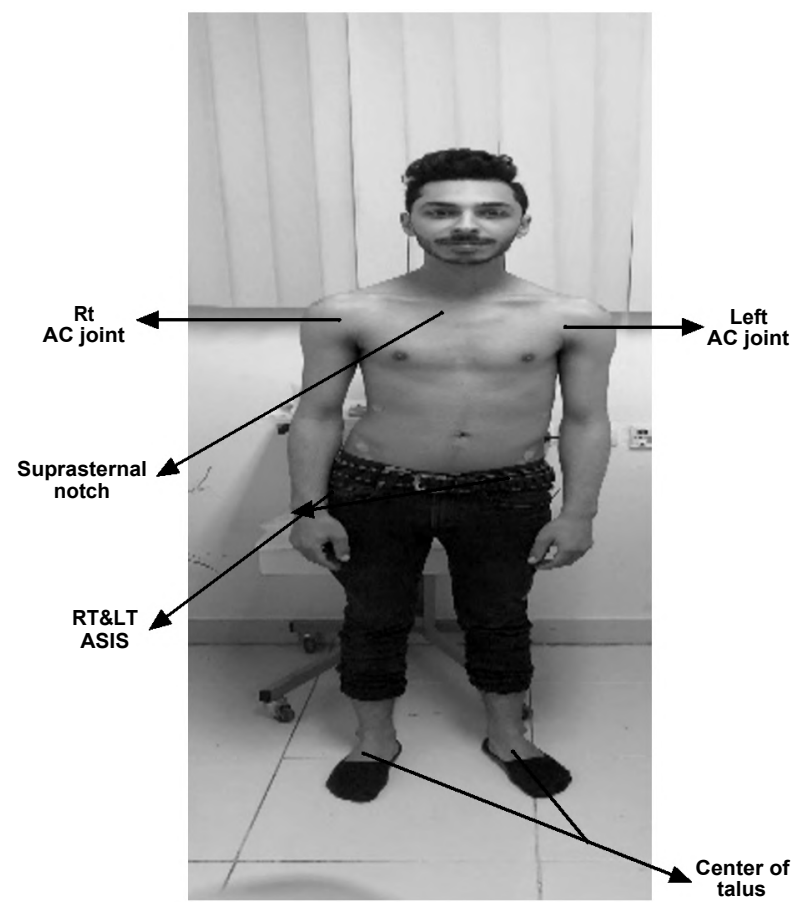

Fig. (2B): Reflective markers from (Anterior view).

For testing validity and (intra) reliability, two photos were taken for each patient form previous views (anterior, lateral, and posterior) by the same examiners on the same day with a resting period of ten minutes between each measure. Following digitization, the PSM application calculates the translations and angulations. displacements. At the end of capturing, the patient sat down and all markers were removed from the body of the subject [7].

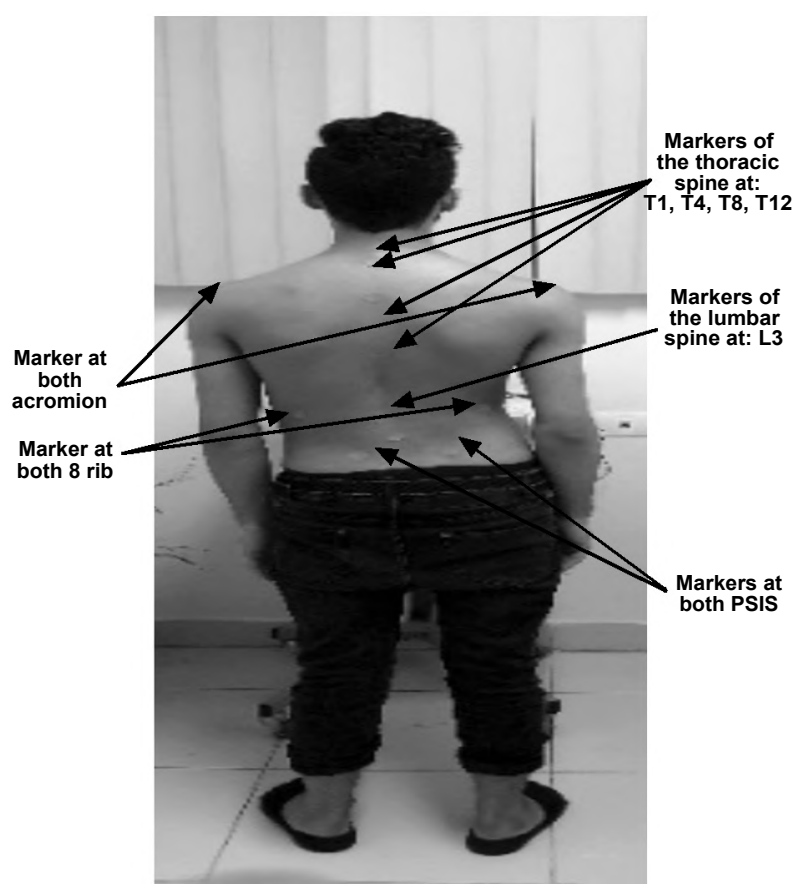

Fig. (2A): Reflective markers (posterior view).

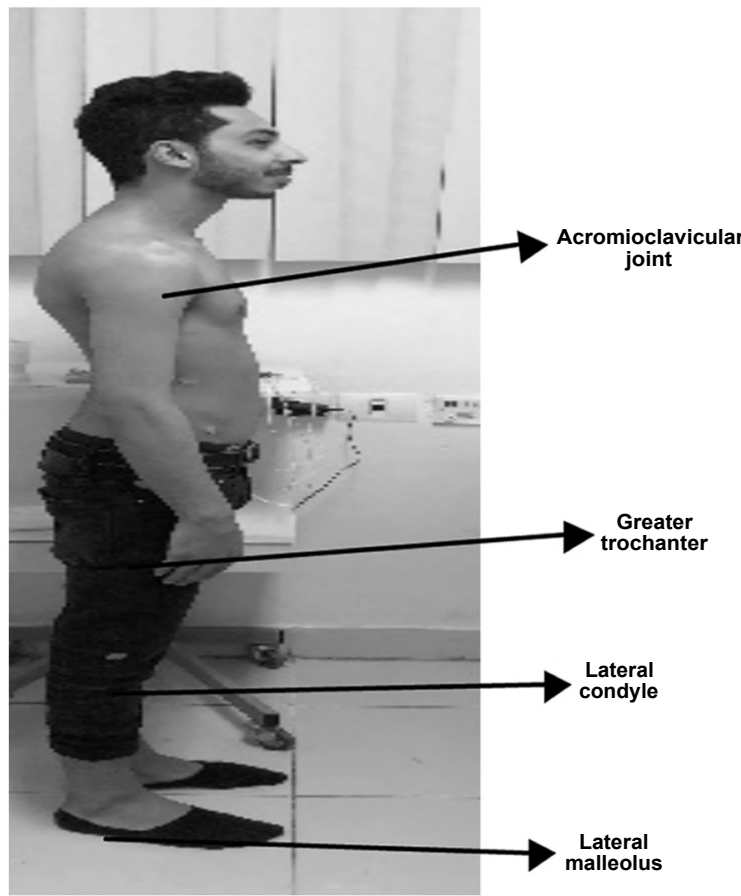

Fig. (2C): Reflective markers (Lateral view).

\section{Results}

Descriptive statistics were carried to calculate the mean \pm SD for all measured variables as shown in Table (1) and Fig. (2). Person Product Moment Correlation Coefficient was conducted to determine the correlation between variables. Intraclass corre- 
lation coefficient (ICCs) with $95 \%$ confidence intervals were conducted for analysis of intra rater reliability. The level of significance for all statistical tests was set at $p<0.05$. All statistical analysis was conducted through the statistical package for social studies (SPSS) version 19 for windows (IBM SPSS, Chicago, IL, USA).

Table (1): Demographic characteristics of the study group.

\begin{tabular}{lllll}
\hline & $\mathrm{X} \pm \mathrm{SD}$ & \multicolumn{3}{c}{ Minimum Maximum Range } \\
\hline Age (years) & $17 \pm 1.2$ & 15 & 19 & 4 \\
Weight $(\mathrm{kg})$ & $65.97 \pm 9.16$ & 50 & 81 & 31 \\
Height $(\mathrm{cm})$ & $168.6 \pm 6.4$ & 155 & 179 & 24 \\
BMI $\left(\mathrm{kg} / \mathrm{m}^{2}\right)$ & $23.1 \pm 2.04$ & 18.37 & 25.51 & 7.14 \\
Cobb's angle & $32.48 \pm 7.8$ & 18 & 45 & 27 \\
$\quad$ (degrees) & & & & \\
Females/males & $28 / 13$ & & & \\
Thoracolumbar/ & $30 / 11$ & & & \\
$\quad$ Lumbar & & & & \\
\hline
\end{tabular}

X : Mean. SD: Standard Deviation.

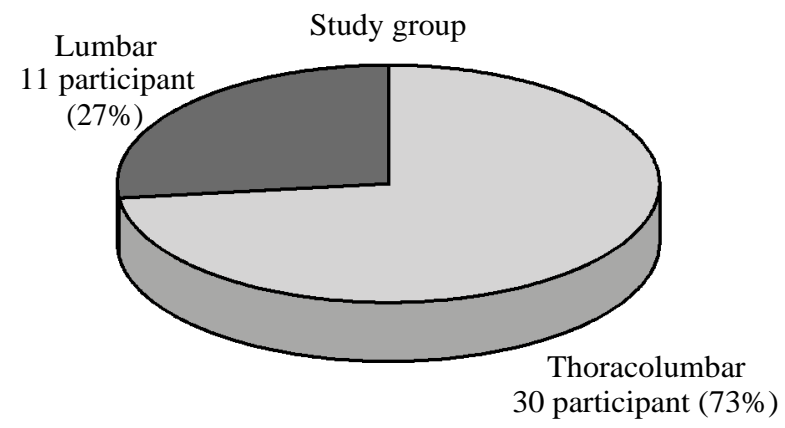

Fig. (3): Scoliotic curve distribution of the study group.

\section{Concurrent validity:}

The correlations between Cobb's angle and segment angulation that measured by posture screen mobile phone were moderate positive significant correlation for T1-T4 ( $r=0.4, p=0.009)$, strong positive significant correlation for T4-T8, T8-T12, T12-L3 and L3-PSIS ( $r=0.82$ to 0.89 ), $p=0.0001$ ) (Table 2 and Figs. 4,5).

Table (2): Correlation between Cobb's angle and segment angulation.

\begin{tabular}{llll}
\hline & $\begin{array}{c}\text { Segment angulation } \\
\text { (degrees) }\end{array}$ & $r$-value & $p$-value \\
\hline Cobb's angle & T 1-T4 & 0.4 & $0.009^{*}$ \\
(degrees) & T4-T8 & 0.86 & $0.0001^{*}$ \\
& T8-T 12 & 0.89 & $0.0001^{*}$ \\
& T12-L3 & 0.83 & $0.0001^{*}$ \\
& L3-PSIS & 0.82 & $0.0001^{*}$ \\
\hline
\end{tabular}

$r$-value: Correlation coefficient value.

$p$-value: Probability value.

*: Significant.

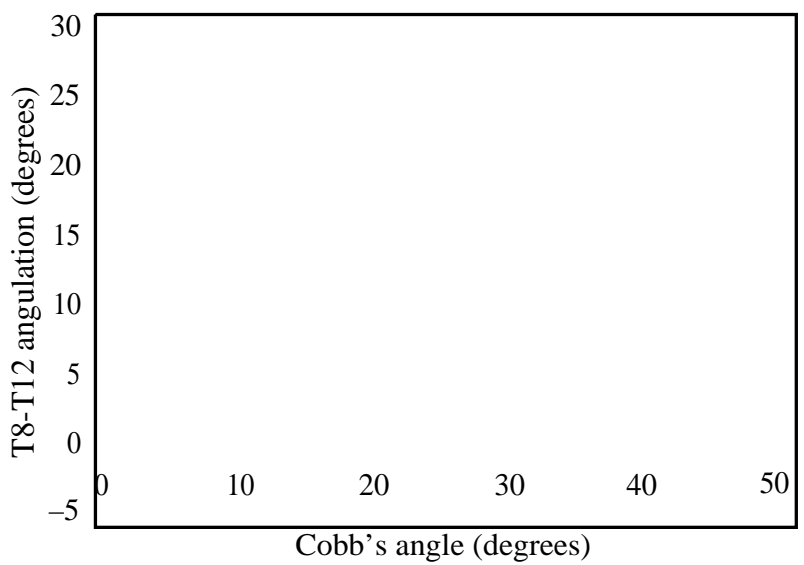

Fig. (4): Correlation between Cobb's angle and T8-T12 segment angulation.

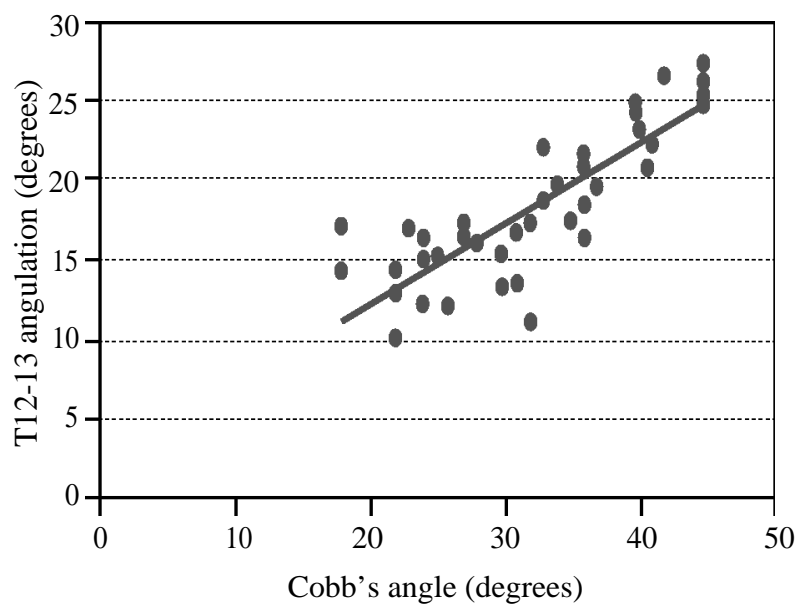

Fig. (5): Correlation between Cobb's angle and T 12-L3 segment angulation.

The correlations between Cobb's angle and segment translation that measured by posture screen mobile phone were moderate positive significant correlation for T1-T4 ( $r=0.6, p=0.009)$, strong positive significant correlation for T4-T8, T8-T12, T12-L3 and L3-PSIS ( $r=0.8-0.9), p=0.0001$ ). (Table 3 and Figs. 6,7).

Table (3): Correlation between Cobb's angle and segment translation.

\begin{tabular}{lccc}
\hline & $\begin{array}{c}\text { Segment translation } \\
(\mathrm{cm})\end{array}$ & $r$-value & $p$-value \\
\hline $\begin{array}{l}\text { Cobb's angle } \\
\text { (degrees) }\end{array}$ & T 1-T4 & 0.6 & $0.0001^{*}$ \\
& T4-T8 & 0.9 & $0.0001^{*}$ \\
& T8-T12 & 0.8 & $0.0001^{*}$ \\
& T12-L3 & 0.85 & $0.0001^{*}$ \\
& L3-PSIS & 0.81 & $0.0001^{*}$ \\
\hline
\end{tabular}

$r$-value: Correlation coefficient value.

$p$-value: Probability value.

*: Significant. 


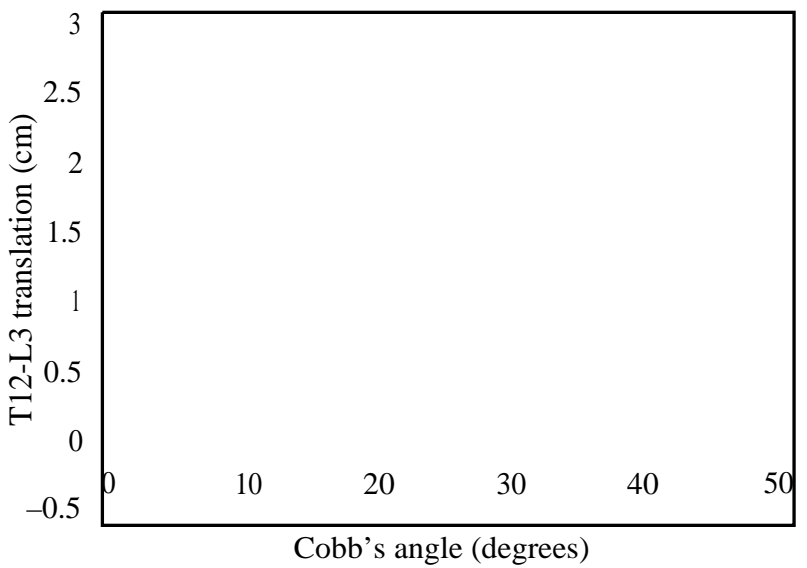

Fig. (6): Correlation between Cobb's angle and T 12-L3 segment translation.

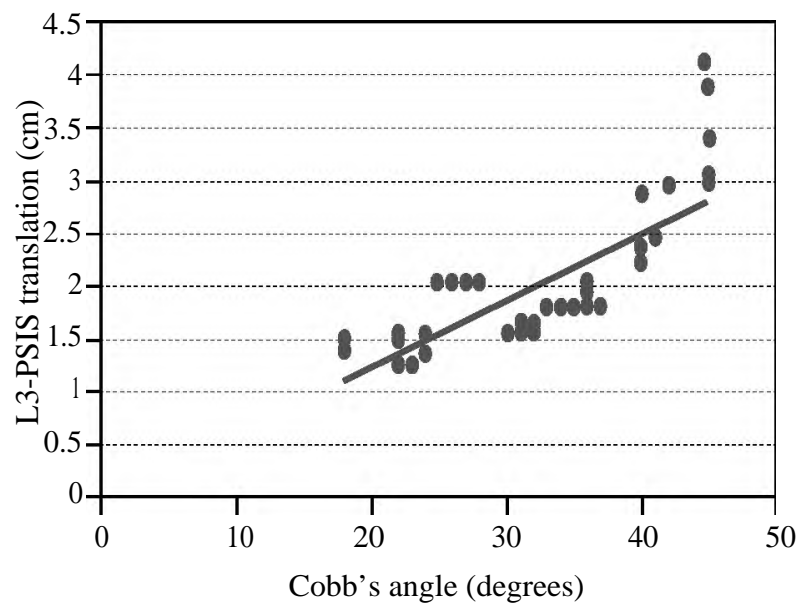

Fig. (7): Correlation between Cobb's angle and L3-PSIS segment translation.

\section{Reliability:}

Intra-rater reliability of the Posture screen mobile phone suggested excellent agreement for segment angulation (ICC: 0.99) and segment translation (ICC: 0.92-0.97) (Tables 4,5).

Table (4): ICC for posture screen mobile phone measurement of segment angulation.

\begin{tabular}{lccc}
\hline \multirow{2}{*}{$\begin{array}{c}\text { Segment angulation } \\
\text { (degrees) }\end{array}$} & ICC & \multicolumn{2}{c}{$(95 \% \mathrm{CI})$} \\
\cline { 3 - 4 } & & Lower bound & Upper bound \\
\hline T 1-T4 & 0.99 & 0.992 & 0.997 \\
T4-T8 & 0.99 & 0.992 & 0.998 \\
T8-T 12 & 0.99 & 0.99 & 1 \\
T12-L3 & 0.99 & 0.993 & 0.998 \\
L3-PSIS & 0.99 & 0.99 & 1 \\
\hline
\end{tabular}

ICC: Inter class correlation coefficient value. CI : Confidence Interval.
Table (5): ICC for posture screen mobile phone measurement of segment translation.

\begin{tabular}{cccc}
\hline \multirow{2}{*}{$\begin{array}{c}\text { Segment translation } \\
(\mathrm{cm})\end{array}$} & ICC & \multicolumn{2}{c}{$(95 \% \mathrm{CI})$} \\
\cline { 3 - 4 } & & Lower bound & Upper bound \\
\hline T1-T4 & 0.95 & 0.91 & 0.97 \\
T4-T8 & 0.92 & 0.87 & 0.95 \\
T8-T12 & 0.96 & 0.94 & 0.98 \\
T 12-L3 & 0.97 & 0.95 & 0.98 \\
L3-PSIS & 0.96 & 0.93 & 0.97 \\
\hline
\end{tabular}

ICC: Inter class correlation coefficient value.

$\mathrm{CI}$ : Confidence Interval.

\section{Discussion}

Increasing the use of smartphone devices among the general population, as well as their high prevalence among medical providers have resulted in the rapid expansion of clinical applications for clinicians [8]. Although, radiographic images allow healthcare professionals to assess the dimensions of the deformity in both the coronal and sagittal planes, they still have a major disadvantage of radiographs which is an increase in the risk of having malignancies with repeated exposure [9] The risk of breast cancer is 4 times greater in these patients [10].

The purpose of this study was to determine the rater reliability and construct validity of a PSM application that was developed for the clinical assessment of posture in AIS. While previous studies have explored the reliability of different posture variations using this application in normal populations and different musculoskeletal problems [11-13], the findings of this study are novel in measuring posture deviation of AIS by unique noninvasive PSM. To our knowledge, this is the first study that has examined both the validity and reliability of PSM application for measuring posture variables in patients with AIS. The studies that were conducted to evaluate the validity and reliability of PSM in measuring postural deviations in normal populations and different musculoskeletal abnormalities were used as a standard reference to our study.

Previous research on the PSM application has found less than desirable inter-and test-retest reliability and notable differences in measurement when compared to a gold standard 3-D system [7] Boland and colleagues (2016) have recently reported ICCs ranged from 0.26 to 0.93 for inter-rater reliability for eleven postural variables (e.g. trans- 
lations \& angulations) using the PSM application [11], while Kan et al. [12] found ICCs ranged from 0.12 to 0.96 for inter-rater reliability across two data collection sessions. Good to excellent intraand inter-rater reliability was obtained when digitizing images using a mobile posture application for both angulations and segment translations. The application also demonstrated construct validity across certain positions.

Hopkins [6] tested the validity and intra-rater agreement of PSM in measuring standing posture by comparing it with the Vicon 3D motion analysis system as the gold-standard measure. He found that the tilt and shift measurements using PSM were repeatedly higher than those measured by the Vicon system in the frontal view while in the lateral view, the results reversed and PSM tended to underestimate tilt and shift measurements. In accordance with the results of our study, Szucs and Donoso Brown [7] conducted a study on twenty healthy young adults to determine rater reliability and construct validity of a PSM. They found that intra-rater reliability ranged from 0.71 to 0.99 and inter-rater reliability was good to excellent for all translations. ICCs were stronger for translations versus angulations. The construct validity analysis found that the application was able to detect the change in the four variables selected. They concluded that PSM has demonstrated strong rater reliability and preliminary evidence of construct validity.

Boland et al. [11] investigated the inter- and intra-rater agreement when using PSM in assessing static standing posture. They performed repeated postural assessments of 10 subjects (minimally clothed or fully clothed) using PSM on two nonconsecutive days. They found that inter-rater agreement was almost perfect $(\mathrm{CCs}>0.81)$ for four measures and substantial $(0.6 \leq \mathrm{CCs}<0.8)$ for three measures during the fully clothed exam, while during the minimally clothed exam, the inter-rater agreement was almost perfect for four measures and substantial for four measures. The Intra-rater agreement between two minimally clothed exams was almost perfect for two measures and substantial for five measures. They concluded that PSM appears to be a reliable easy-use tool for assessing static standing posture and recommend wearing minimal clothing during the assessment. These results are consistent with the results of our study.

Also, Studnicska [13] stated that the PSM application is a useful, simple tool that is easy for dentists to assess the correlation between malocclusion and posture. He found that PSM application might represent a useful instrument in the detection of dental occlusion pathology in the earlier stages. Al-Rawi et al. [14] conducted a study to observe the relationship between musculoskeletal pain (MSP) and vertebral deviations among male dentists in the United Arab Emirates (UAE). PSM application was used to observe postural deviations in the vertebral region. They concluded that Work-related MSP is a significant health issue among dentists and may be associated with vertebral deviation later in life.

Posture screen analysis is an easy, yet objective postural and movement screening instrument that can guide dentists to practice the correct postural exercises. Biomechanics research frequently utilizes electromagnetic tracking sensors or optic sensors with multiple camera systems for data collection methods, however these systems are expensive and may not be an accessible option at smaller academic or research centers. This PSM application presents a cost-effective alternative for both clinics and research centers for measuring and describing baseline posture. The initial cost to purchase the application is minimal in comparison to basic biomechanical tools. While the application costs more than traditional clinical measures of posture, such as goniometers and inclinometers, it offers an analysis in multiple views and increased quantity of variables with additional posture variables not available with clinical instruments (e.g., joint segment translations) [7]

\section{Conclusion:}

Based on the findings of this study, it can be concluded that PSM application is valid and reliable in screening AIS. It is an easy, safe, low-cost, userfriendly, quick, affordable and noninvasive screening tool for patients.

\section{Financial support and sponsorship:}

NIL.

\section{Conflicts of interest:}

There are no conflicts of interest.

\section{References}

1- GOLDBERG C.J., MOORE D.P., FOGARTY E.E. and DOWLING F.E. Scoliosis: A review. Pediatric Surgery International, 24 (2): 129-144, 2008.

2- KONIECZNY M.R., SENYURT H. and KRAUSPE R. Epidemiology of adolescent idiopathic scoliosis. Journal of Children's Orthopaedics, 7 (1): 3-9, 2013.

3- DELORME S., PETIT Y., de GUISE J.A., LABELLE H., AUBIN C.E. and DANSEREAU J.: Assessment of the 3$\mathrm{D}$ reconstruction and high-resolution geometrical modeling of the human skeletal trunk from 2-D radiographic images. 
IEEE Transactions on Biomedical Engineering, 50 (8): 989-998, 2003.

4- ASHER M.A. and BURTON D.C.: Adolescent idiopathic scoliosis: Natural history and long term treatment effects. Scoliosis, 1 (1): 1-10, 2006.

5- ZABJEK K.F., LEROUX M.A., COILLARD C., RIVARD C.H. and PRINCE F.: Evaluation of segmental postural characteristics during quiet standing in control and idiopathic scoliosis patients. Clinical Biomechanics, 20 (5): 483-490, 2005.

6- HOPKINS B.C.B.: Validity of PostureScreen Mobile ${ }^{\circledR}$ in the measurement of standing posture, pp-2-23, 2014.

7- SZUCS K.A. and BROWN, E.V.D.: Rater reliability and construct validity of a mobile application for posture analysis. Journal of Physical Therapy Science, 30 (1): 31-36, 2018.

8- BOULOS M.N.K., WHEELER S., TAVARES C. and JONES R.: How smartphones are changing the face of mobile and participatory healthcare: An overview, with example from eCAALYX. Biomedical Engineering Online, 10 (1): 1-14, 2011.

9- RONCKERS C.M., LAND C.E., MILLER J.S., STOVALL M., LONSTEIN J.E. and DOODY M.M.: Cancer mortality among women frequently exposed to radiographic exam- inations for spinal disorders. Radiation Research, 174 (1): 83-90, 2010.

10- DOODY M.M., LONSTEIN J.E., STOVALL M., HACKER D.G., LUCKYANOV N., LAND C.E. and US Scoliosis Cohort Study Collaborators: Breast cancer mortality after diagnostic radiography: Findings from the US Scoliosis Cohort Study. Spine, 25 (16): 2052-2063, 2000.

11- BOLAND D.M., NEUFELD E.V., RUDDELL J., DOLEZAL B.A. and COOPER C.B.: Inter-and intra-rater agreement of static posture analysis using a mobile application. Journal of Physical Therapy Science, 28 (12): 3398-3402, 2016.

12- KAN S., FORTUNA J. and NUR S.: Can a mobile application be used to assess postural alignment in clinic. Canadian Society for Biomechanics, 12 (5): 82-90, 2016.

13- STUDNICSKA D.: Is PostureScreen ${ }^{\circledR}$ Mobile app an accurate tool for dentists to evaluate the correlation between malocclusion and posture? CRANIO®, 19 (7): 763-66, 2018

14- AL-RAWI N.H., YOUSEF H., KHAMIS M., BELKADI O., AHMED S. and ALI S.: Vertebral Malalignment among Male Dentists with Work-related Musculoskeletal Pain in the United Arab Emirates. The Journal of Contemporary Dental Practice, 19 (7): 773-777, 2018.

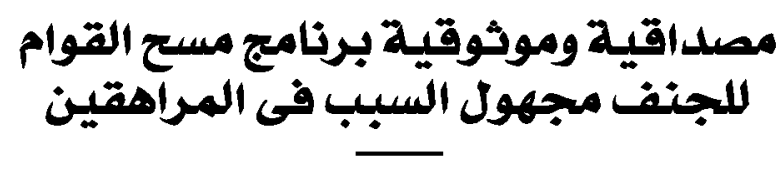

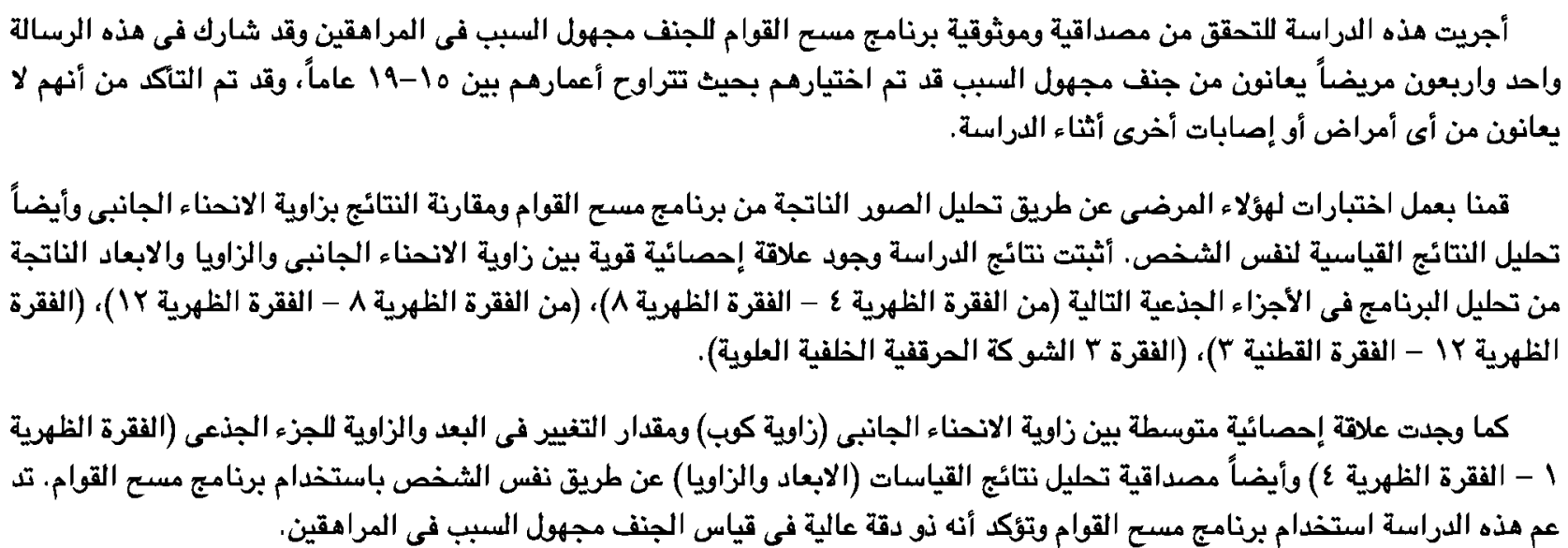

